\title{
INVERTED DECOUPLING 2DOF INTERNAL MODEL CONTROL FOR MIMO PROCESSES
}

\author{
Juwari Purwo Sutikno ${ }^{1 *}$, Zahrotul Azizah ${ }^{1}$, Renanto Handogo ${ }^{1}$, Riza Aris Hikmadiyar ${ }^{1}$, \\ Anwaruddin Hisyam ${ }^{2}$ \\ ${ }^{1}$ Chemical Engineering Department, Institut Teknologi Sepuluh Nopember, Kampus ITS Sukolilo, \\ Surabaya 60111, Indonesia \\ ${ }^{2}$ Faculty of Chemical and Natural Recourses Engineering, Universiti Malaysia Pahang, 26300 \\ Gambang, Kuantan, Malaysia
}

(Received: July 2018 / Revised: October 2018 / Accepted: April 2019)

\begin{abstract}
In general, the multiple-input-multiple-output (MIMO) system is the main method of process control in industry. However, the interaction between variables in the process is a challenge when designing controllers for the system. Strong interaction worsens system performance. Inverted decoupling plays an important role in reducing interaction in the process. Internal model control (IMC) is the controller used in this research. A one degree of freedom (1DoF) IMC controller is only able to provide a good response to set-point tracking, and has a slow response to disturbance rejection. Therefore, a controller that has a good response to set-point tracking and disturbance rejection is a two degrees of freedom (2DoF) IMC. The tuning method uses maximum peak gain margin (Mp-GM) stability criteria based on the uncertainty model. In this study, a reduction in interaction was realized by the addition of inverted decoupling to the 2DoF IMC control scheme. The Wardle \& Wood and Wood \& Berry column distillation models are given as illustrative examples to demonstrate the performance of the inverted decoupling 2DoF IMC control scheme. A comparison is made of the IAE values of 1DoF IMC, $2 \mathrm{DoF}$ IMC, decoupling 2DoF IMC, and inverted decoupling 2DoF IMC, with inverted decoupling 2DoF IMC showing the lowest IAE value.
\end{abstract}

Keywords: Interaction; Inverted decoupling; MIMO system; Mp-GM tuning; 2DoF IMC

\section{INTRODUCTION}

Time delay and coupling are problems that widely occur in industry, especially in the MIMO process (Jin et al., 2016). Coupling is the interaction between process variables and causes difficulties in designing MIMO controllers. One approach to overcoming coupling is by adding additional controllers called decouplers (Seborg et al., 2011). This method is very easy to implement and understand. There are different types of decoupling, such as ideal, simplified, and inverted decoupling, which are often used for industrial process control (Garrido et al., 2014; Li \& Chen, 2014). Ideal decoupling is very easy to use in the design of controllers, but it is rarely employed because it has complicated decoupling elements. Simplified decoupling has a simple decoupler, but the decoupling process is complicated, while inverted decoupling can overcome the weakness of simplified decoupling and achieve the ideal decoupling goals (Chen \& Zhang, 2007).

\footnotetext{
*Corresponding author's email: juwari@chem-eng.its.ac.id, Tel. +62-81-332827554, Fax. 031-599-9282, Permalink/DOI: https://doi.org/10.14716/ijtech.v10i3.2922
} 
Wahid and Ahmad (2016) improved multi-model predictive control, which is used to control the distillation column. This method can reject very large disturbances. The PID controller has been discussed in the literature (Mohebbi \& Hashemi, 2016; Mohebbi \& Hashemi, 2017). However, this has a weakness, which is the presence of new disturbance known after measuring output. Haura et al. (2017) simulated a refinery used oil distillation column using a 1DoF IMC controller, achieving a very good response for set-point change (servo problem). Unfortunately, set-point tracking and load-disturbance rejection in the 1DoF IMC controller scheme cannot be regulated or optimized separately. When the parameters are used for set-point tracking, a slow response to load-disturbance rejection is obtained, and vice versa. This means that it is very difficult to achieve stable and robust control simultaneously between set-point tracking and load-disturbance rejection. Sutikno et al. (2013) developed a 2DoF IMC with the Mp-GM (Maximum peak - Gain Margin) tuning method to obtain IMC control parameters. 2DoF IMC can overcome set-point tracking and load-disturbance rejection separately, without affecting each other. The method was used in the process of containing parametric uncertainty and obtained a very good response. However, the method is still limited to the SISO (Single Input Single Output) system, while processes in industry consist of many variables that interact with each other, so further research into Mp-GM tuning in the MIMO system is needed. Astuti et al. (2015) proposed Mp tuning for the MIMO system that was implemented on the Wood \& Berry distillation column. The response showed good results for set-point tracking, but gave unsatisfactory results when there was a disturbance in the system. Sutikno et al. (2017) proposed a MIMO system able to represent an industrial process called the quadruple tank system. However, interactions between process variables in this system are strong, and an IMC controller with Mp-GM tuning is not fully able to overcome these. This means the MIMO system requires an additional controller to reduce interaction. The purpose of this study is to add additional controllers called inverted decoupling to the 2DoF IMC scheme, with the design objective to reduce interaction significantly. The system used is MIMO $2 \times 2$, which has two inputs and two outputs that interact with each other. The tuning method used in this study is Mp-GM tuning.

\section{METHODS}

\subsection{Two Degrees of Freedom (2DoF) IMC}

A two degrees of freedom controller is a control strategy by which it is possible to design controllers for set-point tracking and disturbance rejection separately (Goodwin et al., 2002). Research on 2DoF controllers has been conducted by Astuti et al. (2015) and Hidayah et al. (2014). A $G c_{1}$ controller (setpoint tracking) has an open loop design, while a $G c_{2}$ (disturbance rejection) controller has a feedback design.

The following are algorithms for $G c_{1}$ and $G c_{2}$ controllers (Sutikno et al., 2013):

$$
\begin{gathered}
G_{c 1}=\frac{1}{k} \frac{\tau s+1}{\lambda_{1} s+1} \\
G_{c 2}=G_{c 1} \frac{\alpha s+1}{\lambda_{2} s+1}
\end{gathered}
$$

where $k$ is the process gain, $\tau$ is the time constant, $\lambda_{1}$ and $\lambda_{2}$ are filter controller parameters, and $\alpha$ is the lead parameter of the $G c_{2}$ controller. Parameter $\lambda_{1}$ is obtained from the tuning results using maximum peak (Mp). Mp is defined as the maximum magnitude of the frequency response of the closed loop system. Because the system uses MIMO, the calculation is made in two steps, the first of which is to calculate $\lambda_{1}$ by assuming a SISO system. 
The SISO equation to calculate $|T(j \omega)|$ is:

$$
\frac{Y_{i}}{Y_{s p_{i}}}=\frac{G c_{i} G p_{i i}}{1+G c_{i}\left(G p_{i i}-G p m_{i i}\right)}, i=1,2
$$

The two results of Equation 3 are included in the following equation. The MIMO $2 \times 2$ equation to calculate $|T(j \omega)|$ is:

$$
\frac{Y_{i}}{Y s p_{i}}=\frac{G c_{i} G p_{i i}+G c_{1} G c_{2}\left(G p_{11} G p_{22}-G p_{12} G p_{21}\right)}{\left(1+G c_{1} G p_{11}\right)\left(1+G c_{2} G p_{22}\right)-G c_{1} G c_{2} G p_{12} G p_{21}}, i=1,2
$$

Parameters $\lambda_{2}$ and $\alpha$ are obtained from the tuning results using gain margin (GM). The $G c_{2}$ controller on each loop has a SISO design with the formula:

$$
G o l_{i}=G c_{i 1} G c_{i 2}\left(G p_{i i}-G p m_{i i}\right), i=1,2
$$

\subsection{Inverted Decoupling $2 \mathrm{DoF}$ IMC}

The decoupling structure on the $2 \times 2$ MIMO system can be seen in Figure 1:

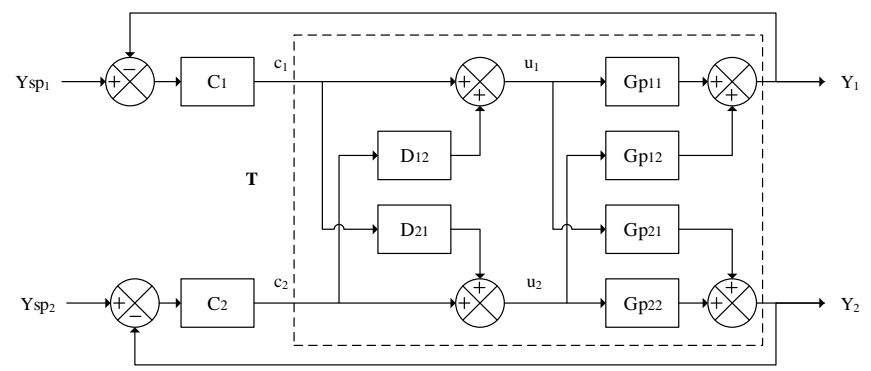

Figure 1 Decoupling structure of $2 \times 2$ MIMO

where $T$ is a decoupled process, $G p$ is the process in the MIMO system, $C$ is the controller, and $D$ is the decoupler, as follows:

$$
\begin{aligned}
& T=\operatorname{diag}\left\{T_{11}, T_{22}\right\} \\
& G p=\left\lfloor G p_{i j,(i, j=1,2)}\right\rfloor_{2 \times 2} \\
& C=\operatorname{diag}\left\{C_{11}, C_{22}\right\} \\
& D=\left\lfloor D_{i j,(i, j=1,2)}\right\rfloor_{2 \times 2}
\end{aligned}
$$

By assuming $D_{11}=D_{22}=1$ in order to simplify the decoupling:

$$
D=\left[\begin{array}{ll}
D_{11} & D_{12} \\
D_{21} & D_{22}
\end{array}\right]=\left[\begin{array}{cc}
1 & -G p_{12} / G p_{11} \\
-G p_{21} / G p_{22} & 1
\end{array}\right]
$$

the decoupled $T$ process becomes:

$$
T=\left[\begin{array}{cc}
T=G p D \\
G p_{11}-\frac{G p_{12} G p_{21}}{G p_{22}} & 0 \\
0 & G p_{22}-\frac{G p_{12} G p_{21}}{G p_{11}}
\end{array}\right]
$$

The results of the $\mathrm{T}$ obtained are complex, meaning the design of the controller for the decoupled process is difficult. Chen and Zhang (2007) proposed an inverted decoupling method 
by modifying the decoupling structure shown in Figure 1 . Assuming $T_{11}=G p_{11}$ and $T_{22}=G p_{22}$, decoupler $D$ then becomes:

$$
\begin{gathered}
D=G p^{-1} T \\
D=\frac{1}{G p_{11} G p_{22}-G p_{12} G p_{21}}\left[\begin{array}{cc}
G p_{22} & -G p_{12} \\
-G p_{21} & G p_{11}
\end{array}\right]\left[\begin{array}{cc}
G p_{11} & 0 \\
0 & G p_{22}
\end{array}\right]
\end{gathered}
$$

From Equation 14, the equation of input process $\mathrm{u}$ to controller output $\mathrm{c}$ becomes :

$$
\left[\begin{array}{l}
u_{1} \\
u_{2}
\end{array}\right]=\left[\begin{array}{cc}
\frac{G p_{11} G p_{22}}{G p_{11} G p_{22}-G p_{12} G p_{21}} & \frac{-G p_{12} G p_{22}}{G p_{11} G p_{22}-G p_{12} G p_{21}} \\
\frac{-G p_{21} G p_{11}}{G p_{11} G p_{22}-G p_{12} G p_{21}} & \frac{G p_{11} G p_{22}}{G p_{11} G p_{22}-G p_{12} G p_{21}}
\end{array}\right] \times\left[\begin{array}{l}
c_{1} \\
c_{2}
\end{array}\right]
$$

If simplified, Equation 15 becomes:

$$
\left[\begin{array}{l}
u_{1} \\
u_{2}
\end{array}\right]=\left[\begin{array}{ll}
1 & 0 \\
0 & 1
\end{array}\right]\left[\begin{array}{l}
c_{1} \\
c_{2}
\end{array}\right]+\left[\begin{array}{cc}
0 & -\frac{G p_{12}}{G p_{11}} \\
\frac{G p_{21}}{G p_{22}} & 0
\end{array}\right]\left[\begin{array}{l}
u_{1} \\
u_{2}
\end{array}\right]
$$

So the inverted decoupling equations become:

$$
\begin{aligned}
& D_{12}=-\frac{G p_{12}}{G p_{11}} \\
& D_{21}=-\frac{G p_{21}}{G p_{22}}
\end{aligned}
$$

The inverted decoupling equation equals the decoupling equation, but the inverted decoupling structure is the opposite of the decoupling structure.

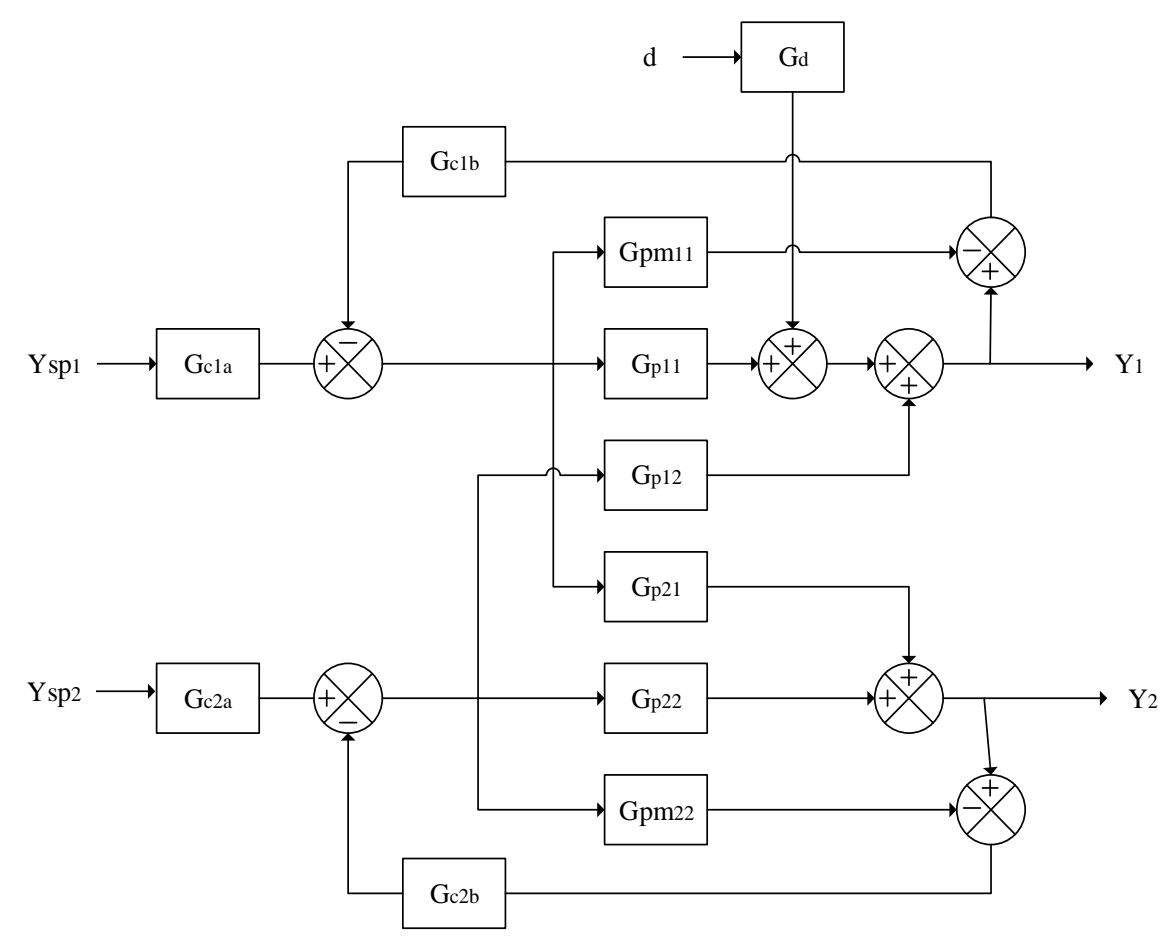

Figure 2 2DoF IMC scheme for 2×2 MIMO system 
Figure 2 shows the $2 \mathrm{DoF}$ IMC scheme without the addition of inverted decoupling for the $2 \times 2$ MIMO system using Mp-GM tuning, while Figure 3 shows a 2DoF IMC scheme with the addition of inverted decoupling for the same system.

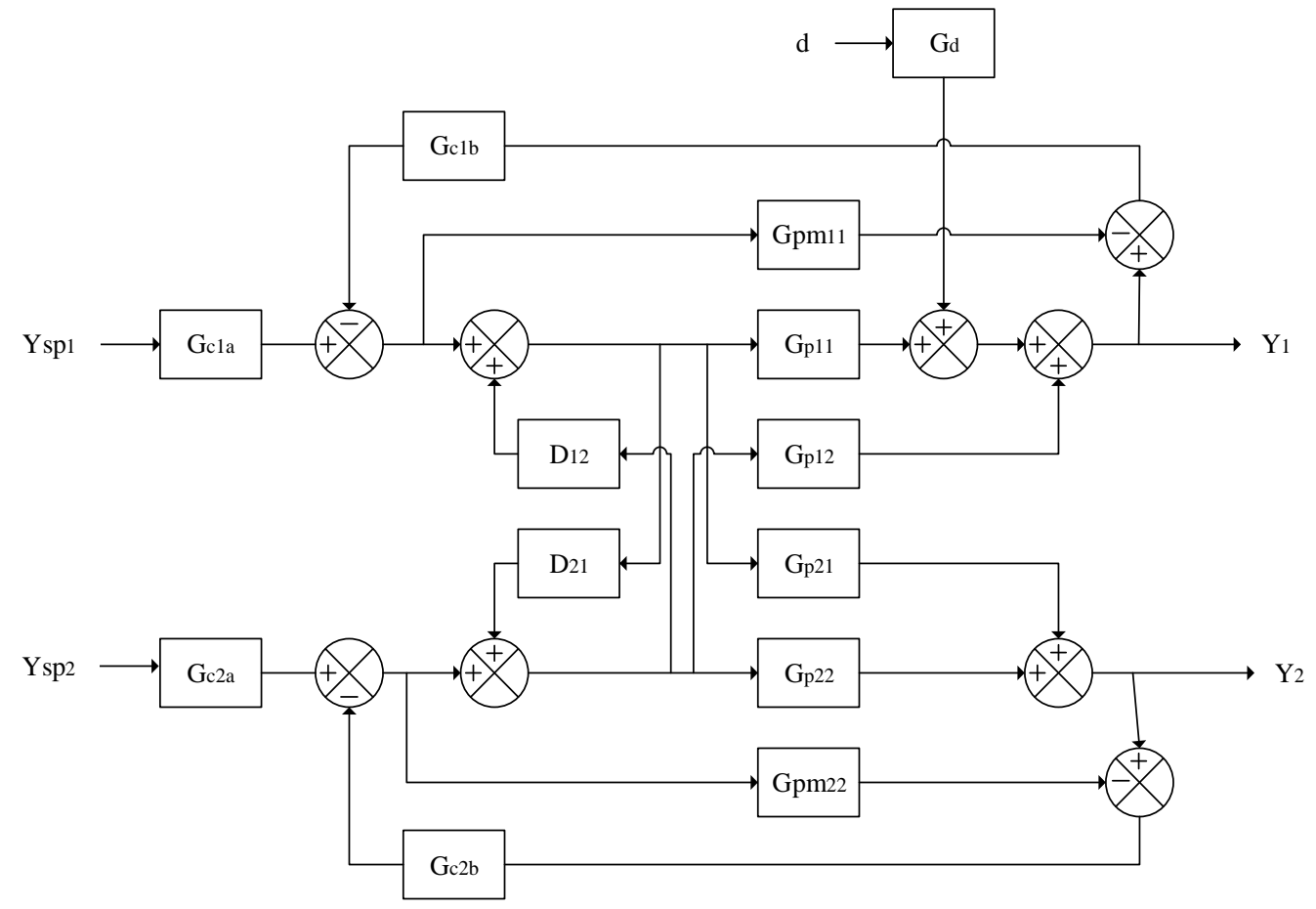

Figure 3 Inverted decoupling 2DoF IMC scheme for $2 \times 2$ MIMO system

\subsection{Experimental Procedure}

The research method consisted of five main steps. The first step was to conduct interaction analysis using the relative gain array (RGA) method in the MIMO $2 \times 2$ system. This method was used to establish the magnitude of the interactions occurring in the MIMO system. The second step was to calculate decoupling based on Equations 17 and 18, while the third step was to determine the worst case based on the uncertainty model. The deviation value was calculated at $\pm 20 \%$ of the model process parameters. The combination of the lower and upper limit parameters was calculated $|T(j \omega)|$ (complementary sensitivity function). The value with the largest max $|T(j \omega)|$ indicated that the most difficult case was controlled. The fourth step was to tune parameter $\lambda_{1}$ using maximum peak (Mp) stability criteria, with the following steps:

1) set the initial value of $\lambda_{1}$ (filter time constant $G c_{1}$ ) equal to $\theta$ (time delay) from the process model, divided by 20 ;

2) determine parameter $\lambda$ on each controller by means of the other controller designed as SISO (Single Input Single Output) to simplify iteration, as in the SISO system there is no interaction between the process variables;

3) calculate the maximum frequency of closed loop frequency response in the frequency range of $\omega=10^{-3}$ to $10^{3}$. Graph used in semilog $|T(j \omega)|$ db vs $\omega$ (frequency);

4) if $\max >1.05$, then $\lambda$ increases by a small amount, for example $\lambda+0.001$.

5) The fifth step was to tune parameter $\lambda_{2}$ (filter time constant $G c_{2}$ ) and $\alpha$ using the stability criterion of gain margin (GM), with the following steps:

i. $\quad$ set the value of $\lambda_{2}$ at less than $\lambda_{1}$. In this research $\lambda_{2}=0.9 \lambda_{1}$;

ii. set the initial value of $\alpha$ equal to $\lambda_{2}$. Add $\alpha$ in small amounts so that the value of GM on the open loop systems is equal to the GM value. A good value of GM is around 1.7-4. 


\section{RESULTS AND DISCUSSION}

Wood \& Berry and Wardle \& Wood distillation columns were used as examples as this study is limited to MIMO $2 \times 2$. The transfer function from both columns was made by interaction analysis to determine the correct pairing configuration with the Relative Gain Array (RGA) method. The results of the interaction analysis with the RGA method show that the diagonal value of the matrix is greater than one and positive, so the pairing used in both columns is 1-1/ 2-2. The decoupling equation on each column was then calculated, with the result showing that both columns are realizable decoupling. The worst case model calculation was then made using MATLAB software.

Table 1 Results of the Wood \& Berry and Wardle \& Wood distillation columns

\begin{tabular}{lcc}
\hline \multicolumn{1}{c}{ Type of Column } & Wood \& Berry & Wardle \& Wood \\
\hline$R G A$ & {$\left[\begin{array}{cc}2.0094 & -1.0094 \\
-1.0094 & 2.0094\end{array}\right]$} & {$\left[\begin{array}{cc}2.6875 & -1.6875 \\
-1.6875 & 2.6875\end{array}\right]$} \\
Decoupling $\left(D_{12}\right)$ & $1.4766 \frac{16.7 \mathrm{~s}+1}{21 \mathrm{~s}+1} e^{-2 \mathrm{~s}}$ & $0.8016 \frac{60 \mathrm{~s}+1}{(48 \mathrm{~s}+1)(45 \mathrm{~s}+1)} e^{-6 \mathrm{~s}}$ \\
Decoupling $\left(D_{21}\right)$ & $0.3402 \frac{14.4 \mathrm{~s}+1}{10.9 \mathrm{~s}+1} e^{-4 \mathrm{~s}}$ & $0.7833 \frac{35 \mathrm{~s}+1}{38 \mathrm{~s}+1}$ \\
Worst case model $\left(G_{11}\right)$ & $\frac{15.36 e^{-1.2 s}}{13.36 s+1}$ & $\frac{0.1512 e^{-7.2 s}}{48 s+1}$ \\
Worst case model $\left(G_{22}\right)$ & $\frac{-23.32 e^{-3.6 s}}{11.52 s+1}$ & $\frac{-0.144 e^{-10 s}}{28 s+1}$ \\
\hline
\end{tabular}

In practice, the model transfer function parameters differ from the process transfer function parameters, so the values of gain, dead time, and the process time constant will deviate. In this study, the deviation value is taken to be approximately $20 \%$. The lower and upper limits of each parameter form a combination of cases. Tables 2 and 3 show the case combination in each loop from the Wood \& Berry and Wardle \& Wood distillation columns. Each loop has eight combinations of process parameters, then the value of $|T(j \omega)|$ is calculated based on Equation 4. The highest $|T(j \omega)|$ value has the most difficult process parameter to control, which is called the worst case. In Table 2, the highest $|T(j \omega)|$ value is in the sixth case. The worst case is then used as a process parameter in the $2 \mathrm{DoF}$ IMC scheme.

Table 2 Case Combination on the Wood \& Berry distillation column

\begin{tabular}{ccccc}
\hline Case & $k$ & $\tau$ & $\theta$ & $\operatorname{Max}|T(j \omega)|$ \\
\hline 1 & 10.24 & 13.36 & 0.8 & 1.0000 \\
2 & 10.24 & 13.36 & 1.2 & 1.0000 \\
3 & 10.24 & 20.04 & 0.8 & 1.0069 \\
4 & 10.24 & 20.04 & 1.2 & 1.0112 \\
5 & 15.36 & 13.36 & 0.8 & 1.0000 \\
6 & $\mathbf{1 5 . 3 6}$ & $\mathbf{1 3 . 3 6}$ & $\mathbf{1 . 2}$ & $\mathbf{1 . 3 9 9 9}$ \\
7 & 15.36 & 20.04 & 0.8 & 1.0081 \\
8 & 15.36 & 20.04 & 1.2 & 1.0160 \\
\hline
\end{tabular}


Table 3 Case combination on the Wardle \& Wood distillation column

\begin{tabular}{ccccc}
\hline Case & $k$ & $\tau$ & $\theta$ & $\operatorname{Max}|T(j \omega)|$ \\
\hline 1 & 0.1 & 48 & 4.8 & 0.9998 \\
2 & 0.1 & 48 & 7.2 & 0.9998 \\
3 & 0.1 & 72 & 4.8 & 1.0062 \\
4 & 0.1 & 72 & 7.2 & 1.0132 \\
5 & 0.15 & 48 & 4.8 & 0.9999 \\
6 & $\mathbf{0 . 1 5}$ & $\mathbf{4 8}$ & $\mathbf{7 . 2}$ & $\mathbf{1 . 3 8 1 0}$ \\
7 & 0.15 & 72 & 4.8 & 1.0099 \\
8 & 0.15 & 72 & 7.2 & 1.0252 \\
\hline
\end{tabular}

The $\lambda$ parameter was then found by using Mp tuning. The calculation was made using MATLAB software. The Mp tuning method determines the controller parameters, so the complementary sensitivity function of each loop can be 1.05 , as at that value the overshoot generated is approximately $10 \%$. If it is less than $10 \%$ the response tends to be slow, but if it is more than $10 \%$ it will tend to trigger instability.

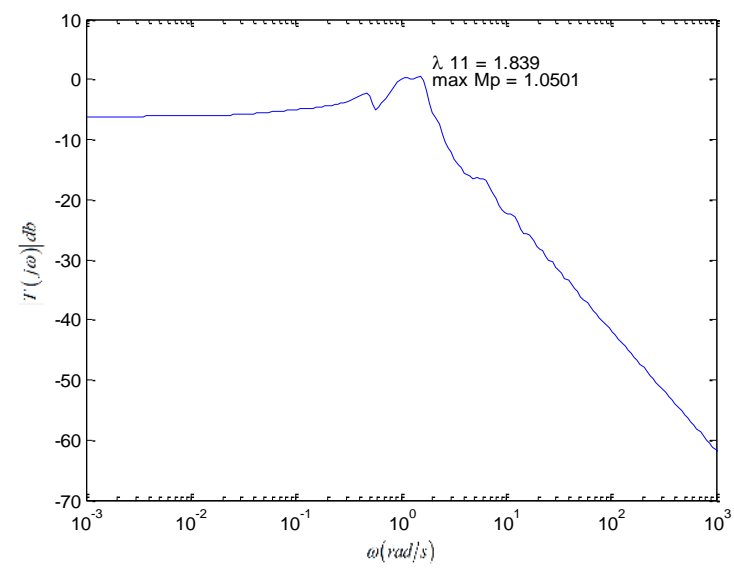

(a)

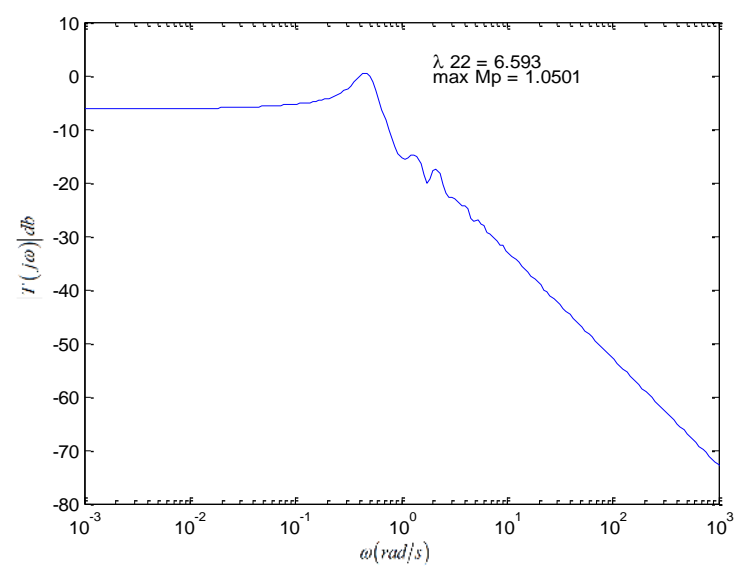

(b)

Figure $4 \lambda_{1}$ parameter results using Mp tuning on the Wood \& Berry column: (a) loop 1; (b) loop 2

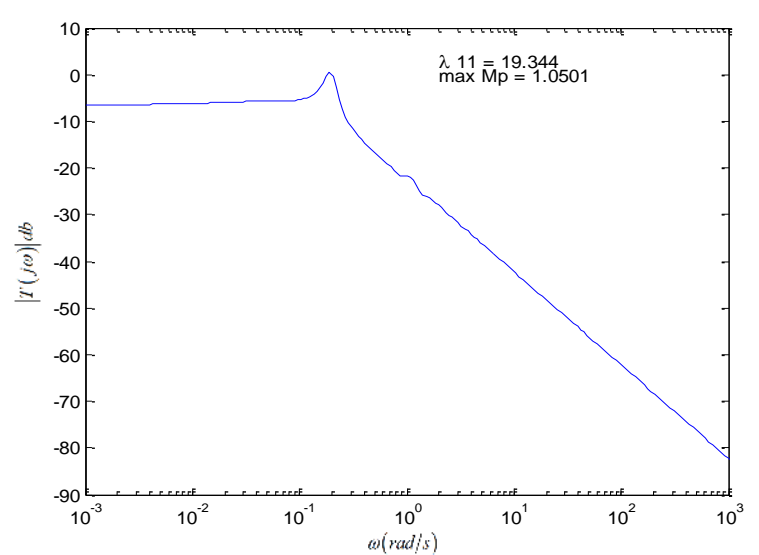

(a)

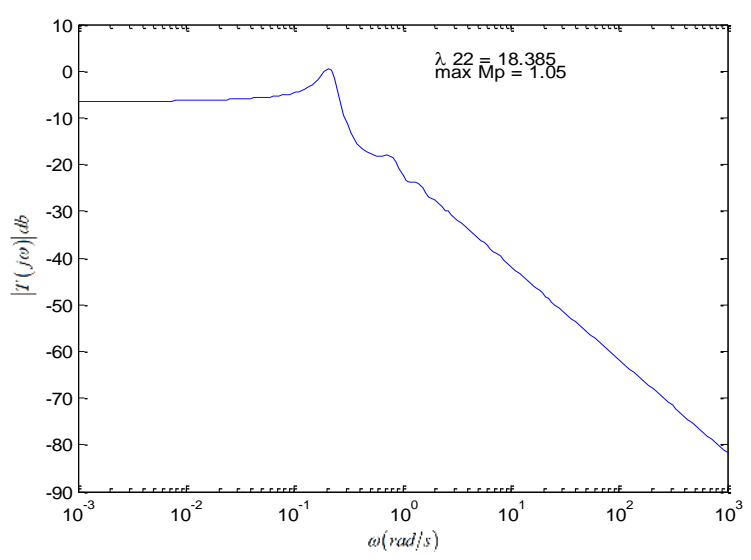

(b)

Figure $5 \lambda_{1}$ parameter results using Mp tuning on the Wardle \& Wood column: (a) loop 1; (b) loop 2 
Parameters $\lambda_{2}$ and $\alpha$ were searched for using GM tuning, with the results on each column as follows.

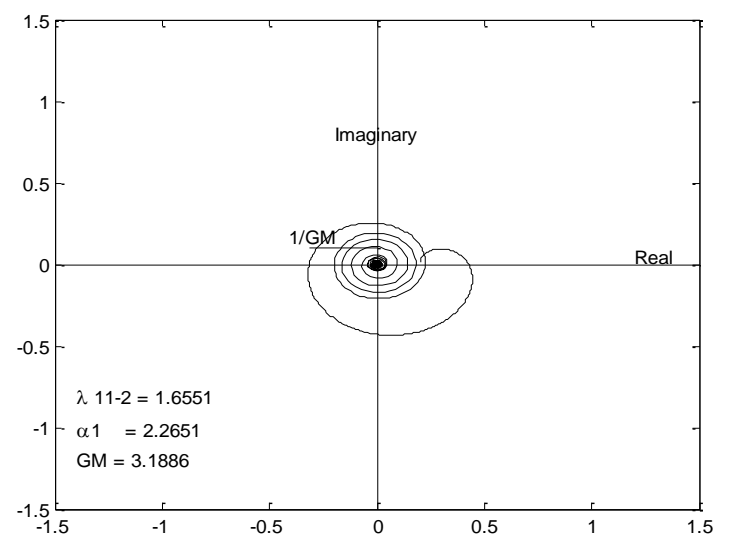

(a)

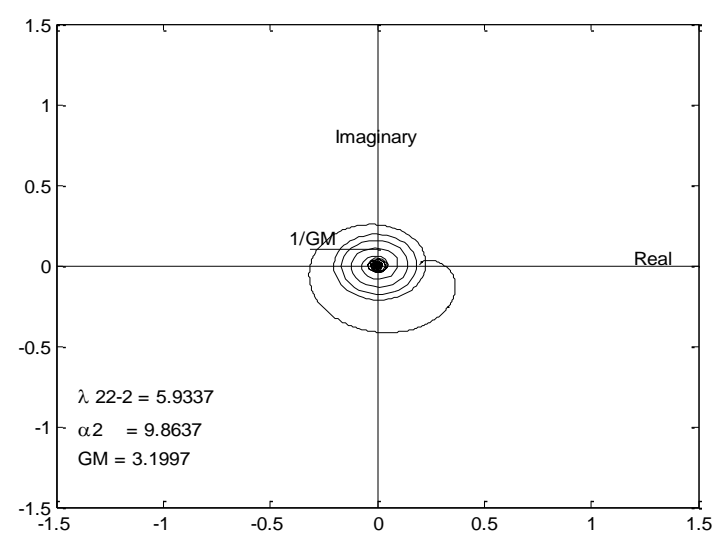

(b)

Figure 6 Results of $\lambda_{2}$ and $\alpha$ parameters using GM tuning on the Wood \& Berry column: (a) loop 1; (b) loop 2

Figure $4 \mathrm{a}$ shows the result of parameter $\lambda_{1}$ on the Wood \& Berry column for loop 1 obtained 1.839 , at a maximum frequency of around 1.5 and maximum peak $(\mathrm{Mp})$ of 1.05 , while Figure $4 \mathrm{~b}$ shows the result of parameter $\lambda_{1}$ for loop 2 obtained 6.593, at a maximum frequency of around 0.5 and $\mathrm{Mp}$ of 1.05 .

Parameter $\lambda_{l}$ on the Wardle \& Wood column is shown in Figures 5a and 5b. Parameter $\lambda_{l}$ for loop 1 obtained 19.344 at a maximum frequency of around 0.2 and $\mathrm{Mp}$ of 1.05 , while parameter $\lambda_{1}$ for loop 2 obtained 18.385, at a maximum frequency of around 0.2 and $\mathrm{Mp}$ of 1.05.

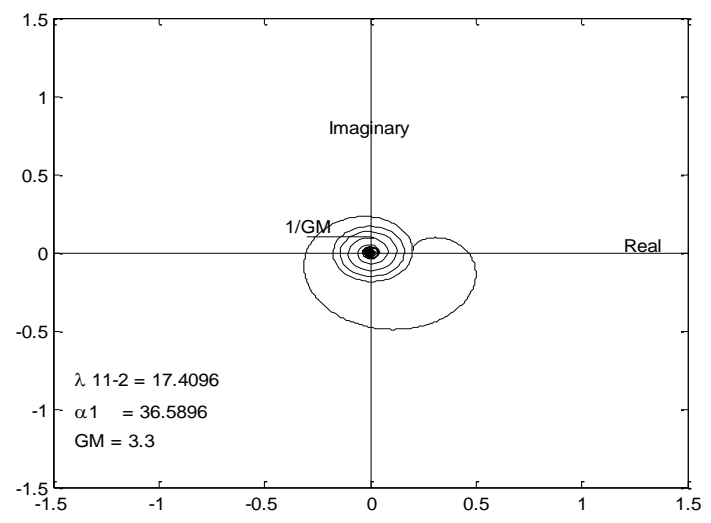

(a)

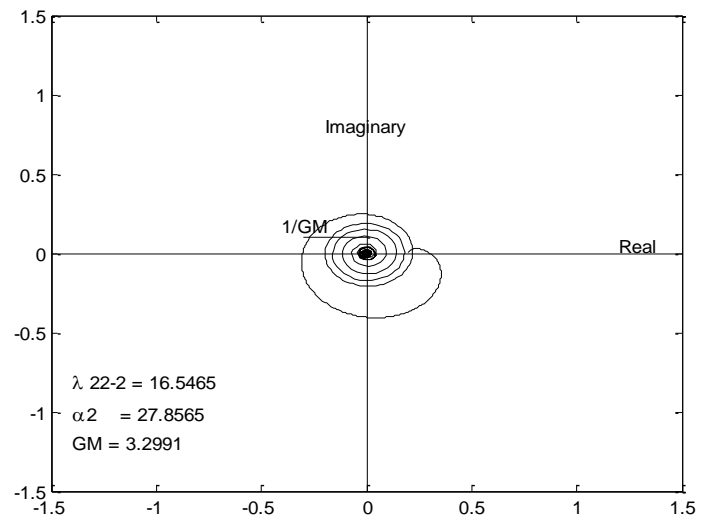

(b)

Figure 7 Results of parameters $\lambda_{2}$ and $\alpha$ using GM tuning on the Wardle \& Wood column: (a) loop 1; (b) loop 2

In Figure 6a, parameters $\lambda_{2}$ and $\alpha$ were obtained at the optimum Gain Margin (GM) of 3.1886. This means that at this value, parameters $\lambda_{2}$ and $\alpha$ gave the smallest IAE (Integral Absolute Error). In Figure 6b, the optimum GM obtained 3.1997. Figure 7 also gives the results of parameters $\lambda_{2}$ and $\alpha$, with the optimum GM in loop 1 being 3.3 and in loop 2 3.2991.

After all the parameters were obtained, a simulation was performed using SIMULINK software for each column. Set-point changes were made in loop 1 and loop 2, and disturbances given in loop 1. 


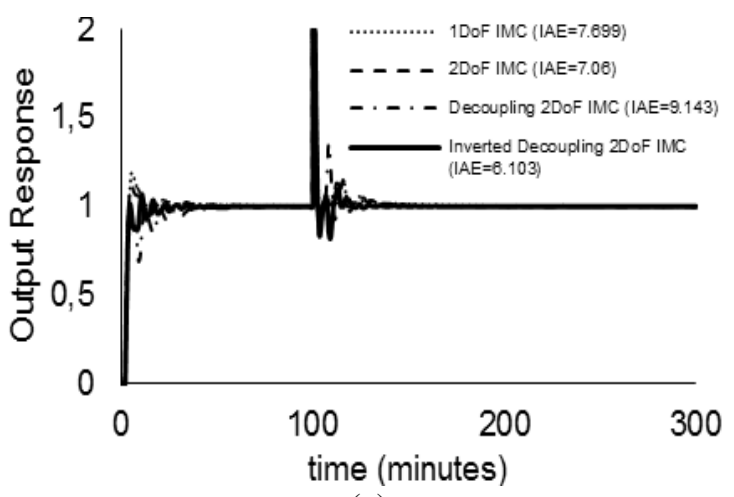

(a)

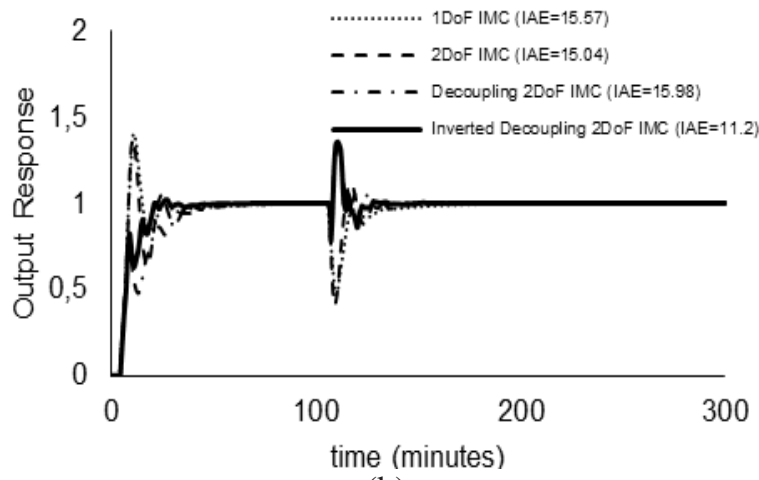

(b)

Figure 8 Response results: (a) loop 1; (b) loop 2 on the Wood \& Berry column

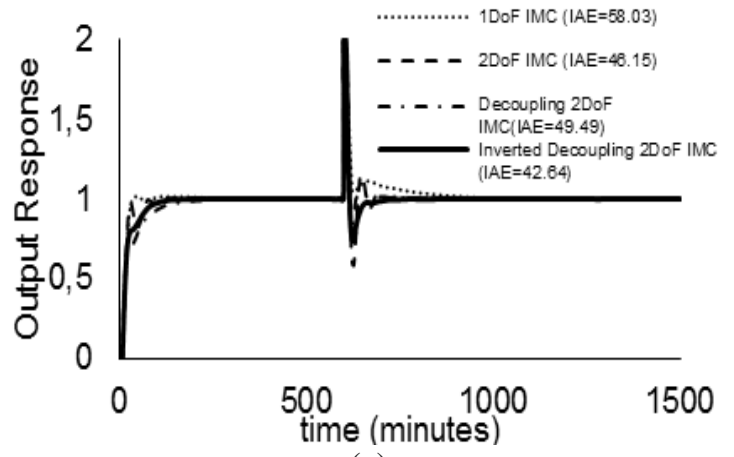

(a)

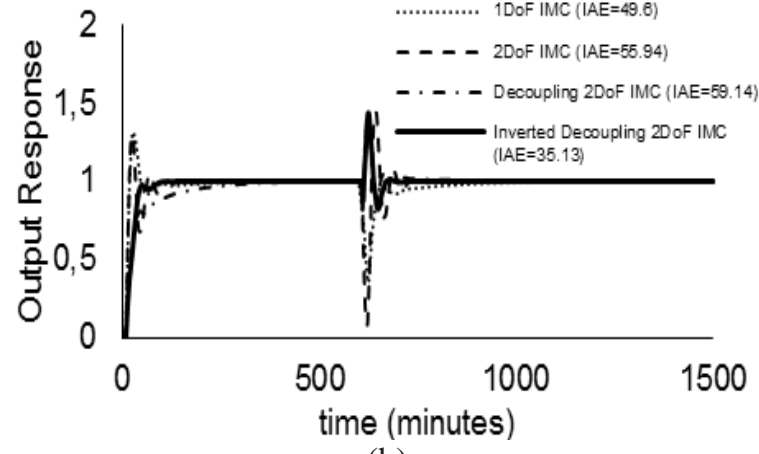

(b)

Figure 9 Response results: (a) loop 1; (b) loop 2 on the Wardle \& Wood column

In this study, Integral Absolute Error (IAE) was used to compare the quality of the controlled responses. A smaller IAE indicates better process control because the optimum settings can minimize the IAE value. In Figures 8 and 9, four controllers are used to compare the results. The first controller uses $1 \mathrm{DoF}$ IMC, and the results give a good response to the setpoint tracking, but a very slow response to disturbance rejection, so the resulting IAE value is still high. IAE integrates the absolute error over time. The IAE value indicates that the lower the IAE value, the better the controller used, and vice versa. The second controller uses 2DoF IMC, with IAE results lower than those of $1 \mathrm{DoF}$ IMC. These results show that GM tuning can overcome the weakness of Mp tuning, which still has a slow response to disturbance rejection. However, in GM tuning the interaction is still high; it can be seen in the simulation results that when there is a disturbance in loop 1, there is still a big change in loop 2. The third controller is 2DoF IMC with the addition of decoupling. Unfortunately, the use of decoupling produces a higher IAE value than without decoupling. The benefit of decoupling may not be fully realized because an imperfect process model or worst case model was used. The fourth controller is $2 \mathrm{DoF}$ IMC with the addition of inverted decoupling; changes in loop 2 tend to be small and not affected by the disturbance in loop 1. From the results of this simulation it can be concluded that 2DoF IMC using Mp-GM tuning with the addition of inverted decoupling gives the smallest IAE value, as inverted decoupling can greatly reduce control loop interactions and the change for one controlled variable has little effect on the other controlled variables.

\section{CONCLUSION}

In this study, the system used was MIMO $2 \times 2$ with Mp-GM tuning. Four controllers were used to compare the results, namely $1 \mathrm{DoF}$ IMC, $2 \mathrm{DoF}$ IMC, decoupling $2 \mathrm{DoF}$ IMC, and inverted decoupling $2 \mathrm{DoF}$ IMC. The results show that inverted decoupling produces the lowest IAE 
value compared to the other controllers. This structure is able to reduce the interaction between variables in the MIMO $2 \times 2$ process.

\section{ACKNOWLEDGEMENT}

Thanks to the Institut Teknologi Sepuluh Nopember (ITS) through its Fresh Graduate Scholarship Program, LPPM ITS, Chemical Engineering Department FTI-ITS, and Process Design and Control Laboratory Chemical Engineering ITS.

\section{REFERENCES}

Astuti, D. W., Juwari, J., Handogo, R., 2015. Mp Tuning for Internal Model Control 2x2 Multi Input Multi Output (MIMO) System. IPTEK Journal of Proceedings Series, Volume 1(1), pp. 467-473

Chen, P., Zhang, W., 2007. Improvement on an inverted decoupling technique for a class of stable linear multivariable processes. ISA Transactions, Volume 46(2), pp. 199-210

Garrido, J., Vázquez, F., Morilla, F., 2014. Inverted decoupling internal model control for square stable multivariable time delay systems. Journal of Process Control, Volume 24(11), pp. 1710-1719

Goodwin, G.C., Graebe, S.F., Salgado, M.E., 2002. Control Systems Design. International Journal of Adaptive Control and Signal Processing, Volume 16(2), pp. 173-174

Haura, A.L., Ma'mun, K.Q., Sutikno, J.P., Handogo, R., 2017. Re-refinery Used Oil Vacuum Distillation Column Control by using Internal Model Control. Chemical Engineering Transactions, Volume 56, pp. 1471-1476

Hidayah, N., Sutikno, J.P., Handogo, R., 2014. Maximum Peak-Gain Margin 2DOF-IMC Tuning for a 2DOF-PID Filter Set Point Controller under Parametric Uncertainty Model. IPTEK Journal of Proceedings Series, Volume 1(1), pp. 8-16

Jin, Q., Du, X., Wang, Q., Liu, L., 2016. Analytical Design 2 DoF IMC Control based on Inverted Decoupling for Non-square Systems with Time Delay. The Canadian Journal of Chemical Engineering, Volume 94(7), pp. 1354-1367

Li, Z., Chen, Y., 2014. Ideal, Simplified and Inverted Decoupling of Fractional Order TITO Processes. In: IFAC Proceedings, Volume 47(3), pp. 2897-2902

Mohebbi, M., Hashemi, M., 2016. Reducing the Vibrations of an Unbalanced Rotary Engine by Active Force Control. International Journal of Technology, Volume 7(1), pp. 141-148

Mohebbi, M., Hashemi, M., 2017. Designing a 2-Degree of Freedom Model of an Unbalanced Engine and Reducing its Vibrations by Active Control. International Journal of Technology, Volume 8(5), pp. 858-866

Seborg, D., Edgar, T., Mellichamp, D., Doyle, F., 2011. Process Dynamics and Control. $3^{\text {rd }}$ Edition. John Wiley \& Sons, Inc.

Sutikno, J.P., Aziz, B.A., Yee, C.S., Mamat, R., 2013. A New Tuning Method for Two-Degreeof-Freedom Internal Model Control under Parametric Uncertainty. Chinese Journal of Chemical Engineering, Volume 21(9), pp. 1030-1037

Sutikno, J.P., Handogo, R., Arifin, I., Rahayu, S.P., Putra, V.D., Fauzan, N., Hisyam, A., 2017. Implementation of State-feedback Controller on Quadruple Tank Modified I System. Chemical Engineering Transactions, Volume 56, pp. 853-858

Wahid, A., Ahmad, A., 2016. Improved Multi-model Predictive Control to Reject Very Large Disturbances on a Distillation Column. International Journal of Technology, Volume 7(6), pp. 962-971 